\title{
Perspectivas de medición orientadas al mercado: revisión metodológica.
}

\author{
SANDRA PATRICIA ROJAS BERRIO ${ }^{1}$
}

\begin{abstract}
RESUMEN
El propósito principal de éste artículo es mostrar las diferentes perspectivas metodológicas de medición orientadas al mercado y proponer un modelo para ello después de revisar la metodología utilizada en distintas aplicaciones empíricas por autores que han investigado el tema.
\end{abstract}

\section{PALABRAS CLAVE}

Orientación al mercado, perspectiva cultural y perspectiva de comportamiento.

\begin{abstract}
The main purpose of this article is to show the different methodology perspectives for measuring market orientation and propose a model for this. First reviewing the methodology used in different empirical applications from who have researched the subject.
\end{abstract}

\section{KEY WORDS}

Market orientation, Perspective and Cultural Perspective behavior.

\section{SOBRE LA ORIENTACIÓN AL MERCADO}

En los últimos años se ha encontrado un renovado énfasis en la entrega a los clientes de productos y servicios de calidad superior ${ }^{2}$ Lo anterior se debe a la necesidad de ajustarse y responder a los constantes cambios del mercado para orientarse más hacia él mismo.

De una manera más formal, y de acuerdo con Kholi y Jaworski, la orientación al mercado se refiere a la gene- ración de inteligencia de mercados en toda la organización dando respuesta pronta a la voz del mercado ${ }^{3}$. Una definición similar plantean Charles H. Noble, Rajiv K. Sinha y Kumar ${ }^{4}$; según ellos, la orientación del mercado es: "el grado en el cual la unidad de negocio obtiene y utiliza la información de clientes, desarrollando una estrategia que resuelve las necesidades del cliente, y los instrumentos por medio de los cuales siempre está la respuesta que los clientes necesitan y desean". Otra definición se encuentra en Day ${ }^{5}$ quien considera el concepto como una exteriorización de procesos organizacionales donde se contrasta el aprendizaje organizacional. Al respecto, Hurley y Hult ${ }^{6}$ tratan la orientación del mercado y las orientaciones de aprendizaje como elementos de la cultura organizacional que influyen en la innovación y en otros resultados. Mientras Slater y Narver ${ }^{7}$ la consideran como efectos de elementos que demuestran el aprendizaje de las organizaciones.

A menos que la dirección no muestre claras intenciones por dar respuesta a las necesidades de los consumidores, la organización no estará orientada al mercado. La respuesta ante cambios en las necesidades de los consumidores, a menudo exige la introducción de nuevos productos o servicios que llevan asociados un alto riesgo para la organización. Si la dirección demuestra un alto grado de aversión al riesgo asociado o al eventual fracaso del producto, es posible que la introducción de nuevos productos que traten de satisfacer las nuevas necesidades aparecidas en el mercado, sea menos probable, con lo que la orientación hacia el consumidor, y por ende el grado de orientación al mercado, sea menor. De ahí se puede afirmar que el grado de aversión al riesgo mostrado por la dirección, es un condicionante del grado de orientación al mercado.

1 Docente-investigadora del Departamento Académico de Mercadeo del Politécnico Grancolombiano. Magister en Administración y Administradora de Empresas de la Universidad Nacional de Colombia. Contacto: srojasbe@poligran.edu.co, sprojasb@gmail.com

2 Bitner 1990; Day and Wensley 1988; Parasuraman, Zeithaml y Berry 1985

3 Kholi y Jaworski 1990

4 Charles H. Noble, Rajiv K. Sinha y Kumar 2002

5 Day 1994.

6 Hurley y Hult 1998.

7 Slater y Narver 1995. 
Partiendo de lo anterior, en la bibliografía revisada se encuentra que la orientación hacia el mercado es un concepto que constituye una de las principales corrientes de investigación en el mercadeo estratégico desarrollado en los últimos veinte años. Desde su creación a principios de 1990, la idea central de la investigación orientada hacia el mercado ha sido la de estudiar la relación entre esta y el rendimiento empresarial ${ }^{8}$.

El dominio específico de la orientación hacia el mercado ha sido objeto de mucho escrutinio en los últimos años. Desde puntos de vista conceptuales y empíricos se han hecho avances para establecer la orientación de mercadeo como la construcción de centrales de las decisiones de comercialización, las actividades empresariales y la cultura de la organización.

Las pocas validaciones empíricas han sido vistas más desde el supuesto de la adopción del concepto de mercadeo, que desde los antecedentes y consecuencias de una orientación hacia el mercado 9 . Un resurgimiento del concepto desde el punto de vista académico y práctico lo retoman autores como Deshpande, Webster, Farley, Houston, Narver, Slater, Olson, Linden y Shapiro ${ }^{10}$.

Teorías recientes y los acercamientos de Zaltman, LeMasters, Heffeing, Coolí y Jaworski definen la orientación al mercado como un conjunto de tres actividades: 1. Generación de inteligencia de mercados en toda la organización de manera que se logre perpetrar en las necesidades futuras de los consumidores. 2. Diseminación de la inteligencia en todas las áreas y departamentos de la organización; y 3. Una amplia sensibilidad y respuesta organizacional a las necesidades de los consumidores.

Sin embargo, sigue siendo una laguna la comprensión de cómo la orientación hacia el mercado puede estar relacionada con la estrategia competitiva. Se argumenta que las actividades orientadas al mercado y los comportamientos se articulan a través de los medios estratégicos que guían a la empresa en su mercado de productos. Conceptualizada como: "orientación estratégica de la empresa comercial", la estrategia competitiva se analiza como un vehículo importante a través de la orientación hacia el mercado, que puede ser manifiesta $^{11}$.

A pesar del creciente interés en este concepto de mercadeo, investigaciones de cómo convertirse en organizaciones más orientadas al mercado son sorprendentemente limitadas. La construcción del concepto de orientación al mercado se puede apoyar metodológica y teóricamente en el de ventaja competitiva ${ }^{12}$.

Algunos autores ven el concepto de la siguiente manera: "La orientación hacia el mercado es una actitud, es decir, un estado de ánimo, no una teoría o una variable operativa. Esta actitud se centra en los estímulos inherentes a la demanda del mercado y la percepción de oportunidades comerciales ${ }^{13}$. Sin embargo más que cualquiera de los conceptos anteriores, es una posición competitiva.

Sin embargo, cabe notar que en la bibliografía revisada los estudios de Homburg Pflesser, Coolí, Jaworski y Kumar $(1993)^{14}$ se encuentran otras propuestas distintas sobre las dimensiones de la orientación hacia el mercado y sus consecuencias.

La investigación sobre el cambio organizacional sugiere que aunque las medidas oficiales son un medio importante de cambio, el proceso de es mucho más complejo. Las organizaciones cambian como resultado de muchas fuerzas, incluidas "las luchas políticas entre facciones dentro de la organización, el cambio evolutivo de la organización a través de la experimentación y el aprendizaje, de las presiones sociales de fuera de la organización, y los cambios que ocurren naturalmente como una empresa madura a través de un ciclo de vida", afirman Zald y Berger ${ }^{15}$.

La orientación hacia el mercado es fundamental para la comercialización de bienes y servicios. Sin embargo para que una organización lo sea más, se deben tener en cuenta factores como los analizados por Gebhardt y Gary F. ${ }^{16}$ quienes desarrollan un modelo teórico para explicar cómo las empresas crearían esa.

\section{Vacíos y hallazgos metodológicos de la orientación al mercado}

A continuación la tabla muestra las investigaciones sobre las dificultades en la ejecución de la orientación al mercado:

8 Chiquan Guo 2002.

9 Barksdale y Darden 1971; HIse 1965; Lusch, Undell y Laczniak 1976; McNamara 1972.

10 Deshpande y Webster 1989; Desphande, Farley y Webster 1993; Houston 1986; Narver y Slater 1990; Olson 1987; Linden 1987;

Shapiro 1988

11 Morgan 1998.

12 Day y Wensley 1983.

13 Lars T. 1990.

14 Homburg Pflesser (2000), Homburg Pflesser (2005), Kohli y Jaworski (1990) y Kohli, Jaworski y Kumar (1993)

15 Zald y Berger 1978.

16 Gebhardt y Gary F 2006 
Tabla 1. Investigaciones sobre dificultades para la ejecución de la orientación al mercado

\begin{tabular}{|c|c|c|}
\hline All'ld & $A \bar{n} \emptyset$ & 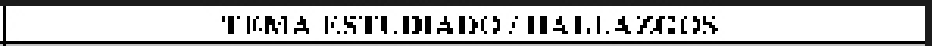 \\
\hline .r.: & 1 ini: & 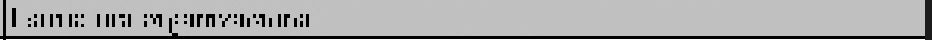 \\
\hline $5 . l_{1} \dot{111}$ & $1: 85$ & 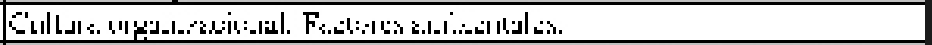 \\
\hline -lessiketr ür & I';:': & 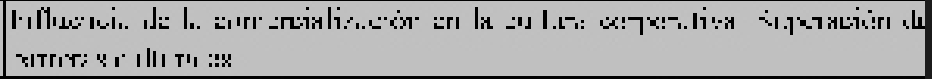 \\
\hline 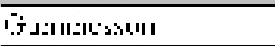 & 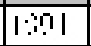 & 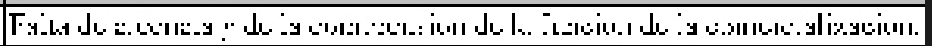 \\
\hline 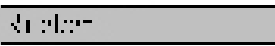 & 15y? & 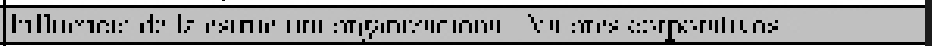 \\
\hline Zı.:k: : & $1 \cdot n_{2}$ & 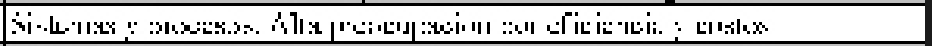 \\
\hline l.ıl l..1 & 154: & 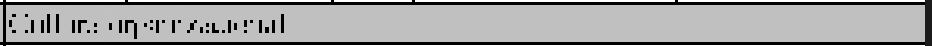 \\
\hline Soiji & $1: 22$ & Col:tlist: in:c.dere.romintr.l \\
\hline 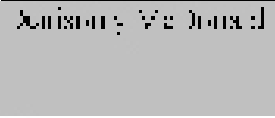 & $1 \% 13$ & 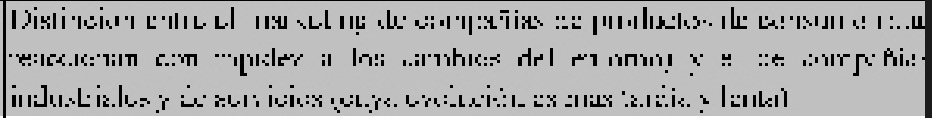 \\
\hline lıтr: & 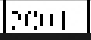 & | \\
\hline
\end{tabular}

Otros atores que han explorado la orientación del mercado desde el punto de vista de su funcionamiento han aplicado una amplia gama de metodologías, de contextos, y de medidas de la orientación ${ }^{17}$. Varios estudios han encontrado la ayuda para la relación fundamental de la orientación y funcionamiento del mercado. Por ejemplo, Pelham ${ }^{18}$ demuestra que la orientación del mercado tiene una relación positiva y significativa sobre una gama de las medidas de funcionamiento, incluyendo eficacia de la comercialización, del crecimiento de las ventas, de la cuota de mercado, y de lo beneficioso. La gama de los resultados positivos asociados a la orientación del mercado ha sido extensa, lo cual se ha demostrado en la relación positiva de los activos $(\mathrm{ROA})^{19}$, el crecimiento de las ventas, el éxito del producto nuevo, y la calidad relativa del producto ${ }^{20}$.

En varios estudios sobre las consecuencias del funcionamiento de la orientación del mercado en los ajustes internacionales ${ }^{21}$, no se ha encontrado ningún efecto, quizás por influencias culturales. Incluso, en diversos contextos, no se ha encontrado ninguna relación directa entre la orientación del mercado y las medidas objetivas de su funcionamiento ${ }^{22}$.

En suma, de la bibliografía revisada con respecto a los inhibidores se pueden extraer tres grandes conclusiones; primero, los empleados, sus opiniones, actitudes, creencias y valores compartidos son un factor determinante de los altos niveles en la orientación hacia el mercado. Segundo, las innovaciones e invenciones de los empleados, en tecnología, estructuras, procesos y estrategias también son factores importantes en el desarrollo y mantenimiento de la orientación al mercado. En tercer lugar, el inhibidor letal para la orientación al mercado son aquellas características internas de la organización que no permiten escuchar la voz del mercado.

\section{Componentes internos de la orientación al mercado}

Diferentes autores encuentran que la creación de una orientación hacia el mercado requiere cambios en la cultura de una organización y la creación de una organización que comparta lo que comprende del mercado con todas las áreas y niveles jerárquicos.

Luego deben existir ciertos antecedentes para que dicha orientación hacia el mercado exista. El primer antecedente pertenece a la alta gerencia de una organización, quien tiene un rol crítico en la fundamentación de los valores y en la orientación de una institución ${ }^{23}$. Esta debe motivar a los individuos en la organización para encaminarla al cambio de mercados, compartir lo que arroja el sistema de inteligencia de mercados con otras áreas y tener respuesta a las necesidades del mercado.

En cuanto a la postura frente al riesgo, la alta gerencia debe responder a las necesidades cambiantes del mercado; lo que a menudo requiere la introducción de nuevos productos y servicios para ir junto con la evolución de las expectativas y necesidades de los consumidores. Pero los nuevos productos, servicios y programas con-

17 Deshpandé 1999.

18 Pelham 2000.

19 Narver y el pizarrero 1990

20 Pelham y Wilson 1996

21 Bhuian 1998

22 Han, Kim, y Srivastava 1998

23 Felton 1959; Hambrick y Mason 1984, Webster. 
llevan un nivel de riesgo porque pueden fallar y tienden a tornarse más importantes que los establecidos. Si la alta gerencia demuestra voluntad para tomar riesgos y fracasos ocasionales como un proceso natural, los mandos medios dan más ideas para introducir nuevas ofertas en respuesta a los cambios en las necesidades de los consumidores. En contraste, cuando la alta gerencia posee aversión al riesgo y es intolerante al fracaso, los subordinados se encontrarán menos atentos a generar y diseminar la inteligencia de mercados o a responder a los cambios en las necesidades de los consumidores.

Además, la postura de la gerencia debe tener los siguientes componentes: agresividad ${ }^{24}$, análisis ${ }^{25}$, defen siva $^{26}$, proyeccion $^{27}$, proactividad ${ }^{28}$ y riesgo ${ }^{29}$.

El segundo antecedente son las dinámicas interdepartamentales, específicamente, la resolución de conflictos de manera coherente y la conectividad y flujo de información entre ellos. En dichas dinámicas existen relaciones de orden-desorden (institutivo)-(instituyente) ${ }^{30}$.

Lo anterior depende intrínsecamente del tercer antecedente que es la existencia de sistemas gerenciales de información con las características de formalidad y centralización. Dicho sistema requiere procesos de investigación, análsis, diseño, implementación y mantenimiento que se ajusten a las necesidades y al presupuesto de la organización. En ellos debe participar toda la organización con el fin de que la información funcione para todos los departamentos. y dé soporte a los objetivos y estrategias de la organización.

Tal sistema de información debe proporcionar, a todos los niveles de la organización, la información necesaria para controlar las actividades y ser adaptable a la evolución de la organización.

Sin embargo, para la consolidación de los anteriores antecedentes existen obstáculos culturales que, como resultado, inhiben el desarrollo de la orientación al mercado. Existe gran cantidad de estudios sobre las barreras realcionadas con el tema. Los siguientes párrafos narrarán los hallazgos al respecto.

\section{Inhibidores y barreras a la orientación ha- cia el mercado}

Las investigaciones sobre las barreras a la orientación de mercado pueden ser divididas en dos aspectos: esporádicas y holísticas ${ }^{31}$. Aunque estas últimas no son excluyentes para Harris ${ }^{32}$ quien trata de explicar el tema desde la articulación que han hecho sobre otros autores.

Las investigaciones esporádicas sobre los inhibidores de la orientación al mercado, período que este autor data a partir de 1950 hasta hoy, se caracterizan por intentos individuales para identificar los obstáculos a la orientación hacia el mercado. Por otro lado, las investigaciones holísticas presentan una conciencia académica por su interés en estudiar el fenómeno.

\section{Componentes externos: construcción de preferencias de los consumidores y orien- tación al mercado}

Teniendo en cuenta que la orientación al mercado debe controlar los inhibidores estudiados en la bibliografía mencionada, que por otro lado tiene tres componentes internos: la alta gerencia, las dinámicas interdepartamentales y los sistemas de información, es muy importante tener en cuenta aquellos elementos externos que constituyen las preferencias de los consumidores.

Indudablemente las empresas deben adaptar su estructura organizativa hacia el mercado para entender la construcción de preferencias de los consumidores y así, encontrarse con resultados financieros acordes con sus objetivos estratégicos. Los autores Kohli y Jaworski $^{33}$ han estudiado las consecuencias del diseño organizacional sobre la orientación y posteriormente en el rendimiento. Atendiendo a lo anterior, la medición del nivel de orientación al mercado se realiza a partir de la comparación de las preferencias del consumidor -construidas a partir de parámetros lógicos y pertinentes al mercadeo- con los productos y servicios ofrecidos por la empresa.

Los parámetros por medio de los cuales se construyen y se hallan las preferencias de los consumidores son, por un lado, el bien o servicio considerado desde la necesidad a satisfacer pasando por su componente real, hasta aquellas características de producto aumentado como garantía, soporte y servicio al cliente.

31 Harris 1996

32 Harris

33 Kohli y Jaworski 1990 
Por otro, las formas de distribución ideales para el individuo teniendo en cuenta situaciones ideales para el mismo. Y la comunicación, puesto que esta es la forma como los individuos pueden llegar a enterarse del producto.

Por último, el precio, teniendo en cuenta en este punto, las formas de pago deseadas. En suma estos parámetros constituyen el valor real que genera el bien o servicio en el consumidor. La siguiente figura ilustra el modelo propuesto para la construcción de preferencias:

Figura 1. Modelo de construcción de preferencias

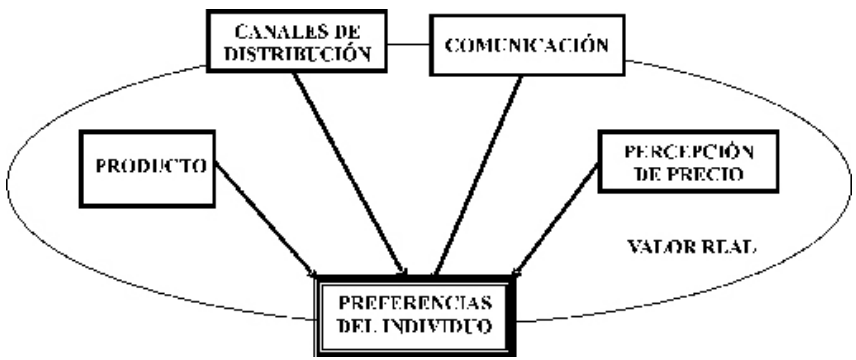

Sin embargo, cabe notar que las empresas que salen al mercado más tarde, poseen barreras de entrada derivadas de la posición preferente y de los costos de cambio llamada "ventaja pionera" ${ }^{34}$. En estos casos, los autores Carpenter y Nakamoto sostienen que de la ventaja pionera puede surgir del proceso por el cual los consumidores aprenden acerca de las marcas y la forma de sus preferencias.

Este proceso de aprendizaje y construcción de preferencias puede producir una estructura que favorece a los primeros entrantes en el mercado, lo que hace difícil para los que ingresan más tarde a competir, puesto que el pionero poseerá una gran cuota de mercado. Esta situación se da incluso si el reposicionamiento de las marcas y los costos de cambio son mínimos.

En muchos mercados, a lo largo de los años, siempre hay rivales de las marcas y mercados que perduran más que otros; una de las razones es por lo que aquí se ha llamado ventaja pionera, y es lo que más domina en el mercado después de la introducción de un nuevo producto. Ser pionero genera una ventaja frente a las otras marcas, y de esta forma hace más difícil el ingreso a nuevos competidores. Pero para ello es importante tener en cuenta las características de los productos, la calidad y sobre todo, el respaldo que genera la misma marca.

34 CARPENTER, NAKAMOTO: 2000

En cuanto a las marcas, el consumidor desarrolla cierto conocimiento y aprende cuáles son sus ventajas y beneficios, luego construye sus preferencias gracias a la adopción de un producto determinado. De lo anterior se deduce que durante el proceso de aprendizaje se produce una ventaja competitiva que de manera positiva el consumidor la considera como la perfecta combinación de atributos: El "pionero" recibe la fuerza de asociación dentro de una categoría de productos.

Las diferencias en participación de mercado son amplias especialmente para las marcas que entran temprano, llamadas "pioneras en el mercado" o "primeros entrantes". Esta ventaja es notable en varios aspectos. Aparece resistente a las acciones de los competidores, sobreviviendo a la introducción de nuevas marcas, a la innovación que realizan los rivales existentes, a la competencia de precios de genéricos o de importaciones y al desplazamiento de los gustos de los consumidores.

\section{Modelo y variables planteadas para el aná- lisis de la orientación al mercado.}

De toda la bibliografía revisada se concluye que la orientación al mercado, desde el punto de vista de la empresa, se compone de dos grandes factores,. El primero es relativo a los procesos internos de cada organización; es decir a la perspectiva cultural de la organización. El segundo, a la construcción de preferencias de los consumidores; o perspectiva de desempeño. El siguiente gráfico ilustra los componentes de la orientación al mercado y constituye el modelo para el análisis en esta investigación.

\section{Figura 2. Componentes de la orientación al mercado}

FACTORES INTERNOS - P. CULTURAL

DESEMPEÑO

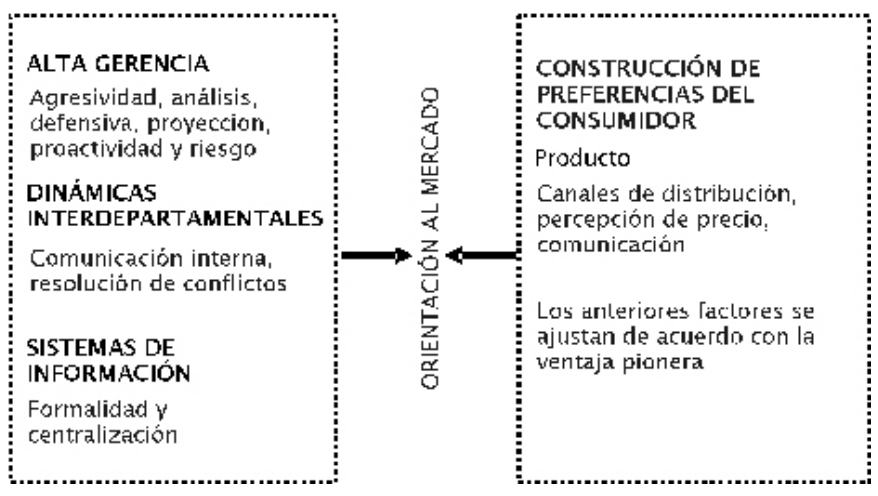


A continuación una tabla con las variables a tratar:

Tabla 2. Variables

\begin{tabular}{|c|c|}
\hline \multicolumn{2}{|r|}{ FACTORES INTERNOS } \\
\hline & ALTA GERENCIA \\
\hline$\sqrt{1}$ & Posic on frente a riesgo \\
\hline W2 & Postura en el mercads \\
\hline$\sqrt{3}$ & $\begin{array}{l}\text { DINAMICAS INTERDEPARTAMENTALES } \\
\text { Nivel de combunicación interna }\end{array}$ \\
\hline V4 & $\begin{array}{l}\text { Capacidad pera la resolución de conflictos } \\
\text { SISTEMAS DE INFORMACION } \\
\text { Formalidad del sistema ce infomacionn }\end{array}$ \\
\hline$\sqrt{6}$ & Nivel de centralicabion dél sistema de información \\
\hline \multicolumn{2}{|r|}{ FACTORES EXTERNOS } \\
\hline & PRODUCTO \\
\hline$\sqrt{7}$ & Percepcion de cal dad \\
\hline v8 & Nivel de sa:išacción del sliente con el producto \\
\hline Wa & $\begin{array}{l}\text { Cantidad de dïerenciales ofrecidas percioidas } \\
\text { rea roenta }\end{array}$ \\
\hline$\sqrt{10}$ & $\begin{array}{l}\text { CANALES DE DISTRIBUCIÓN } \\
\text { Cantidad de Ganales }\end{array}$ \\
\hline $\begin{array}{l}v 11 \\
v 12 \\
v 13\end{array}$ & $\begin{array}{l}\text { Nivel Je sa:Is'acción con respecto a canales de } \\
\text { distribucion } \\
\text { Nivel Je sa:is‘acción de las integrantes del canal con } \\
\text { las utilidëdes } \\
\text { PERCEPCION DE PRECIO } \\
\text { COMUNICACIÓN }\end{array}$ \\
\hline$\sqrt{14}$ & Formas de comunicacior preferidas por los clientes \\
\hline
\end{tabular}

\section{CONCLUSIONES}

La investigación sobre el tema: orientación a mercado se ha desarrollado a partir de dos grandes perspectivas que han permitido trabajar este concepto. Por un lado, se encuentra una perspectiva cultural de la organización. Y por otro lado, se tiene un punto de vista táctico que refiere el comportamiento o desempeño de la organización frente al mercado dando cuenta de las acciones y de la puesta en práctica de las estrategias planteadas por la alta gerencia de la empresa.

En las validaciones empíricas se encuentra que las metodologías empleadas sólo exploran la filosofía y el pensamiento de la empresa que tiende a creerse orientada al mercado, dejando de un lado los fundamentos del concepto. Por lo tanto es imposible mirar la brecha - nivel de orientación al mercado - que existe entre lo ofrecido y lo que realmente espera el mercado. Lo anterior debe hacerse sin olvidar la importancia de los as- pectos internos de la organización, dado que ellos son absolutamente influyentes en el proceso de orientación al mercado.

El concepto de orientación al mercado no debe construirse ni medirse únicamente desde una de las perspectivas, dado que ello lo haría parcializado e incompleto. Por lo anterior, el modelo propuesto permite ver tanto la actitud y cultura de la organización frente al mercado como la brecha que hay entre lo ofrecido y lo recibido por los consumidores.

En suma el modelo permite medir de una manera cuantitativa el nivel de orientación al mercado y de una manera cualitativa la actitud de la empresa frente a la orientación al mercado. 


\section{BIBLIOGRAFÍA}

-Bitner, Mary Jo. "Evaluating Service Encounters: The Effects of Physical Surroundings and Employee Responses”, en Journal of Marketing, Vol. 54, abril, 1990, pág.69-82.

-Day, G.S. and Wensley, R. "Assessing advantage: a framework for diagnosing competitive superiority", en Journal of Marketing, Vol. 52, abril, 1988, pág. 1-20

-Deshpande, R. y Webster, F.E. "Jr. Organizational Culture and Marketing: Defining the Research Agenda”, en Journal of Marketing, Vol. 53, enero, 1989. pág. 3-15. -Houston, Franklin S. "The Marketing Concept: What It Is and What It Is Not", en Journal of Marketing, Vol. 50, abril, 1986.pág. 81-87.

-Olson, David. "When Consumer Firms Develop a Marketing Orientation" [conferencia], Cambridge: 1987.

-Shapiro, Benson P."What the Hell is "Market Oriented'?", en Boston, MA: Harvard Business Review, núm. 66, noviembre-diciembre, 1988, pág. 19-25.

-Kohli, A. K. y Jaworski, B.J. "Market orientation: The construct, research propositions, and managerial implications", en Journal of Marketing. núm.54, 1990, pág.1-18.

-Day, George S. y Wensley, Robin. "Marketing Theory with a Strategic Orientation", en Journal of Marketing, Vol. 47, otoño, 1983. pág.79-89.

-Berry, L; Parasuraman, A. y Zeithaml, V.A. "A Conceptual Model of Service Quality and Its Implications for Future Research," en Journal of Marketing, Vol.49, otoño, 1985. pág.41-50

-Day, George S. "Continuous Learning about Markets", en California Management Review, forthcoming, Vol. 20, pág. 47-49

-Hise, Richard T. "Have Manufacturing Firms Adopted the Marketing Concept?", en Journal of Marketing, Vol.29, julio,1965, pág. 9-12.

-McNamara, Carlton P. "The Present Status of the Marketing Concept”, en Journal of Marketing, Vol. 36, enero, 1972, pág. 50-57.

-Houston, Franklin S. "The Marketing Concept: What It Is What It Is Not", en Journal of Marketing. Vol. 50, abril, 1986, pág. 81-87.

-Lusch, R.; Udell, J.G. y Laczniak, G.R. "The Practice of Business", en Business Horizons, núm. 19, diciembre, 1976, pág. 65-74.

-Deshpande, R.; Farley, J.U. y Webster, F.E. “Corporate Culture, Customer Orientation, and Innovativeness in Japanese Firms: A Quadrad Analysis", en Journal of Marketing, Vol. 57, enero, pág. 23-37.
-Deshpande, R. y Webster, F.E. "Organizational Culture and Marketing: Defining the Research Agenda”, en Journal of Marketing, Vol. 53, enero, 1989, pág.3-15.

-Narver, J.C. y Slater, S.F. "The Effect of a Market Orientation on Business Profitability", en Journal of Marketing, Vol. 54, octubre, 1990, pág. 20-35.

-Olson,D."When Consumer Firms Develop a Marketing Orientation" [conferencia], Cambridge, abril, 1987

-Linden, William J. "Marketing Marketing: The Ryder System Story" [conferencia], Cambridge, abril, 1987. -Shapiro, Benson P. "What the Hell is 'Market Oriented'?", en Boston: Harvard Business Review, núm. 66, noviembre-diciembre, 1988, pág. 119-35.

-Zaltman G., LeMasters, K. y Heffring, M. Theory Construction in Marketing. New York: John Wiley and Sons, Inc., 1982.

-Kohli, A.K. y Jaworski, B.J. "Market Orientation: The Construct, Research Propositions, and Managerial Implications”, en: Journal of Marketing, núm. 54, abril, 1990, pág. 1-18.

-Day, G.S. y Wensley, R., "Marketing Theory with a Strategic Orientation", en Journal of Marketing, núm. 47, 1983, pág. 79-89.

-Pelham, A.M. y Wilson, D.T. "A longitudinal study of the impact of market structure, firm structure, strategy and market orientation culture on dimensions of small firm performance", en Journal of the Academy of Marketing Science, vol. 24 No. 1, 1996, pág. 27-43.

-Felton, A.P. "Marketing the marketing concept work", en Boston: Harvard Business Review. Vol. 37, 1959, pág.55-65.

-Hambrick, D.C. y Mason, P.A. "Upper Echelons: The Organization as a Reflection of Its Top Managers", en Academy of Management Review, núm. 9, 1984, pág. 193-206.

-Venkatraman, N. "Strategic orientation of business enterprises: the construct, dimensionality and measurement”, en Management Science, Vol. 35, núm.8,1989, pág. 942-962.

-Manu, F.A. y Sriram, V. "Innovation, marketing strategy, environment and performance", en Journal of Business Research, Vol. 35, 1996, pág. 79-91. 